Linguistique, littérature, didactique

\title{
Réemploi et déformation du déjà-là dans La Jalousie d'Alain Robbe-Grillet
}

Repetition, variation, distortion in Jealousy by Alain Robbe-Grillet

\section{Catherine Fuchs}

\section{(2) OpenEdition}

Journals

\section{Édition électronique}

URL : http://journals.openedition.org/pratiques/3277

DOI : $10.4000 /$ pratiques.3277

ISSN : 2425-2042

\section{Éditeur}

Centre de recherche sur les médiations (CREM)

Référence électronique

Catherine Fuchs, « Réemploi et déformation du déjà-là dans La Jalousie d'Alain Robbe-Grillet », Pratiques [En ligne], 173-174 | 2017, mis en ligne le 10 mars 2017, consulté le 19 avril 2019. URL: http://journals.openedition.org/pratiques/3277 ; DOI : 10.4000/pratiques.3277

Ce document a été généré automatiquement le 19 avril 2019

(c) Tous droits réservés 


\title{
Réemploi et déformation du déjà-là dans La Jalousie d'Alain Robbe-Grillet
}

\author{
Repetition, variation, distortion in Jealousy by Alain Robbe-Grillet
}

\section{Catherine Fuchs}

1 La présente étude est consacrée aux mécanismes d'autoreformulation à l'œuvre dans $L a$ Jalousie d'A. Robbe-Grillet, roman paru en 1957 aux éditions de Minuit. Il s'agira de décrire les microvariations de forme observables dans les reformulations multiplement réitérées (et chaque fois subtilement déformées) de certaines séquences textuelles ${ }^{1}$ et de s'interroger sur les objectifs poursuivis par l'auteur à travers cette stratégie particulière d'écriture.

2 La Jalousie s'inscrit dans le cadre du huis-clos d'une habitation coloniale (maison, terrasse et jardin), où un mari jaloux, narrateur invisible et in-nommé, épie - notamment à travers les lattes ajourées des volets, d'où la polysémie du titre en français - sa femme, désignée par la simple initiale A..., et Franck, qu'il soupçonne d'être l'amant de A...

Conformément à l'esthétique du "Nouveau Roman», les descriptions minutieuses du narrateur invisible restituent « la présence opaque des choses » (selon l'expression même d'A. Robbe-Grillet, 2013, p. 77). Au fil du texte, confronté à une série de tableaux perçus et/ou remémorés par le jaloux, le lecteur soucieux de cohérence s'interroge sur la topographie des lieux, les déplacements de l'observateur au sein de l'habitation, la chronologie des faits.

4 Le roman repose sur un petit nombre de scènes liées à quelques motifs obsessionnels, révélateurs de la jalousie du mari, qui reviennent de façon récurrente tout au long du texte. Parmi ces derniers, citons le motif de la lettre: le mari voit que A... tire de la commode de la chambre une lettre (qui a été pliée en quatre), puis qu'elle en écrit une autre dont elle efface un mot et qui se retrouve ensuite, visible (bien que pliée en huit), dans la poche de Franck. Citons également le motif de la tache, liée au motif précédent : de l'évocation de l'action d'effacer un mot (à l'aide d'une gomme et d'une lame de rasoir), on glisse à l'action d'effacer (à l'aide des mêmes instruments) une tache sur le mur provoquée par Franck écrasant une scutigère ${ }^{2}$, ce qui contribue à installer la thématique 
obsessionnelle de la tache à laver. De proche en proche, c'est tout un réseau d'associations d'idées qui se trouve ainsi mis en place, tournant autour de l'acte sexuel (redouté/imaginé par le mari jaloux) entre les deux amants: les doigts crispés de la femme sur la nappe du dîner lors de l'écrasement de la scutigère deviennent, dans les fantasmes du jaloux, les doigts crispés sur un drap, les mouvements de la scutigère traquée évoquent ceux de la copulation, etc.

Notre étude portera sur deux scènes emblématiques, qui permettent d'éclairer certaines caractéristiques du processus d'écriture mis en œuvre. Pour la commodité du travail, nous avons considéré les 9 sections du texte ${ }^{3}$ comme autant de chapitres (que nous avons numérotés de 1 à 9) et avons numéroté les paragraphes du texte au sein de chacun de ces 9 chapitres. Chaque exemple sera donc suivi de l'indication du chapitre et du numéro de paragraphe ainsi que de la référence de la page où il figure (dans l'édition originale).

\section{La scène du pont}

6 La première scène que nous étudierons est celle qui évoque le travail d'ouvriers réparant un pont. Cette scène est observée par le mari depuis la terrasse, lorsqu'il suit le regard d'A... dirigé vers le fond de la vallée. La scène se trouve décrite principalement au Ch. 5 dans cinq passages distincts, non contigus, entrecoupés par l'irruption d'autres scènes et de motifs divers.

7 Comme nous allons le voir, ces passages se présentent comme des variations au sein desquelles répétitions et déformations se mêlent de façon indissociable pour créer des suites d'images dont l'ordonnancement chronologique et le statut de réalité ne laissent pas d'être problématiques pour le lecteur.

\subsection{La chronologie des étapes de la réparation du pont}

Les différents passages du Ch. 5 consacrés à cette scène contiennent quelques séquences quasi-synonymes (dans le fond du vallon/dans le fond de la vallée; le pont de rondins qui franchit la petite rivière/le pont en rondins/le petit pont sur le ruisseau) qui garantissent la stabilité du cadrage spatial de la scène. Ils comportent également un certain nombre d'indications aspectuo-temporelles variées (en train de, s'apprêtent à, encore, vont, ne ... plus, a disparu, a fait son apparition, toujours, il reste, etc.), corroborées par des indications topologiques extrêmement précises sur la disposition (à chaque stade) des cinq ouvriers et celle des cinq troncs de remplacement, qui invitent le lecteur à reconstruire la chronologie suivante :

- Première étape : les préparatifs, avant le début des travaux (les cinq troncs neufs en travers du chemin ; deux ouvriers sur la rive droite, trois sur la rive gauche).

(1) Dans le fond du vallon, des manœuvres sont en train de réparer le pont de rondins qui franchit la petite rivière. Ils ont enlevé le revêtement de terre sur un quart environ de la largeur. Ils s'apprêtent à remplacer les bois envahis de termites par des troncs neufs, [...], qui gisent en travers du chemin d'accès, juste avant le pont. [...].

Les deux premiers bois se sont placés parallèlement l'un à l'autre (et à la rive), l'espace entre eux équivalant au double environ de leur diamètre commun. Un troisième les coupe en biais vers le tiers de leur longueur. Le suivant, perpendiculaire à celui-ci, bute contre son extrémité [...]. [Le] cinquième rondin est 
encore parallèle aux deux premiers, ainsi qu'à la direction du ruisseau sur lequel est bâti le petit pont. (Ch. 5, § 9-10, p. 102-103)

(2) Les ouvriers du pont sont au nombre de cinq, comme les troncs de rechange. Ils sont en ce moment tous accroupis dans la même position [...]. Ils sont disposés face à face, deux sur la rive droite, trois sur la rive gauche. Ils discutent sans doute de la façon dont ils vont s'y prendre pour accomplir l'opération, ou bien se reposent un peu avant l'effort, [...]. (Ch. 5, § 13-15, p. 103-105)

- Deuxième étape : un état d'avancement des travaux où l'un des rondins a été remplacé (quatre troncs neufs sur la rive droite, un tronc usé sur la rive gauche; un ouvrier sur la rive droite, quatre sur la rive gauche).

(3) Tout en bas, dans le fond de la vallée, la disposition des personnages n'est plus la même, de part et d'autre du pont en rondins. Il ne reste qu'un seul des ouvriers sur la rive droite, les quatre autres étant alignés en face de lui. Mais leur posture, à aucun d'eux, n'a changé. Derrière l'isolé, un des bois neufs a disparu : celui qui en chevauchait deux autres. Un tronc à l'écorce terreuse, en revanche, a fait son apparition sur la rive gauche, nettement en arrière des quatre ouvriers qui regardent vers la maison. (Ch.5, § 28, p. 107-108)

- Troisième étape : un état ultérieur d'avancement des travaux (sans doute observé à deux moments différents), où trois rondins ont été remplacés (deux troncs neufs en travers du chemin; d'abord un ouvrier sur la rive droite, deux sur la rive gauche, et deux sur le pont ; puis deux sur la rive droite, deux sur la rive gauche et un sur le pont).

(4) [...] puis le pont qui franchit la petite rivière, où les cinq hommes accroupis sont maintenant disposés de la façon suivante: un sur la rive droite, deux sur la rive gauche, deux autres sur le tablier lui-même, près de son bord aval ; [...].

Il ne reste plus que deux bois neufs à placer. (Ch. 5, § 32, p. 109)

(5) La limite aval de ce trapèze est soulignée par la présence du chemin d'accès qui aboutit au petit pont sur le ruisseau. Les cinq hommes y sont maintenant ordonnés en quinconce, deux sur chaque berge et un au milieu, accroupi, [...].

Sur la rive droite il reste toujours deux troncs neufs à placer [...] en travers du chemin qui remonte vers le jardin et la maison. (Ch.5, § 54-55, p. 118)

12 La scène du pont ne constitue pas (comme on pourrait le penser à première vue) une digression superflue, sans rapport avec le huis-clos entre les trois personnages. En effet, dans chacun des passages ci-dessus, le tableau correspondant de la scène surgit suite à un détournement du regard du mari. Celui-ci tourne ses yeux vers le pont au fond de la vallée visible depuis la terrasse où il se trouve, afin de ne pas être surpris en train d'épier A... (seule dans la chambre ou en compagnie de son supposé amant sur la terrasse lors d'un apéritif).

Ces différents tableaux, entrecoupés de façon «stroboscopique " par divers autres fragments de texte, peuvent donc être lus comme une succession d'étapes dans les travaux, perçues par le mari chaque fois qu'il regarde dans cette direction. Mais cette séquence temporelle se révèle fragile. Chacune des étapes, qui se présente comme un plan fixe, un arrêt sur image, est référée à un instant (maintenant, en ce moment) dont rien ne permet de déterminer l'ancrage effectif dans un référentiel externe, tel qu'on pourrait le faire dans le cadre d'un récit linéaire.

14 Par ailleurs, les tableaux du Ch. 5 contrastent avec d'autres tableaux de la scène du pont (aux Ch. 6 et 7), de nature à déconcerter un lecteur avide de repères stables :

(6) L'œil maintenant ne discerne plus rien, malgré les fenêtres ouvertes.

Les cinq ouvriers sont toujours à leur poste, dans le fond de la vallée, accroupis en quinconce sur le petit pont. L'eau courante du ruisseau scintille encore des derniers reflets de la pénombre. Et puis, plus rien. (Ch. 6, $\S 48-49$, p. 138) 
Le passage (6) ne correspond à aucune des étapes décrites au Ch. 5 : l'indication selon laquelle les cinq ouvriers sont accroupis en quinconce sur le petit pont diffère à la fois de (2) où ils sont tous accroupis mais pas en quinconce, et postés sur chacune des deux rives et non pas sur le pont - et de (5) - où ils sont en quinconce mais où un seul est accroupi et posté sur le pont ! L'incohérence de ce tableau est manifeste puisque, dans la pénombre, la scène ne peut plus être perçue par l'observateur (l'œil ne discerne plus rien).

(7) [...] le sol scintille des innombrables toiles chargées de rosée, que les araignées minuscules ont tendues entre les mottes. Tout en bas, sur le pont de bois qui franchit la petite rivière, une équipe de cinq manœuvres s'apprête à remplacer les rondins dont les termites ont miné l'intérieur. (Ch. 7, § 92, p. 176-177) début des travaux : les variations synonymiques entre (7) et (1) sont en effet minimales ( pont de bois/pont de rondins; remplacer les rondins/remplacer les bois; dont les termites ont miné l'intérieur/envahis de termites).

Or, dans (6) et (7), la scène est observée par le mari, respectivement le soir (pénombre) et le matin (rosée) - deux périodes qui, dans les deux chapitres concernés, entourent la nuit d'angoisse où, resté seul (il n'est plus en présence de A... et de Franck), il attend en vain leur retour du voyage et se met progressivement à délirer : les évocations des faits et gestes d'A..., puis de A... en photo, se mêlent indissociablement, entraînant à leur suite ces retours à la scène du pont. L'ancrage effectif dans la réalité devient donc problématique : plutôt que de perceptions hic et nunc, il s'agit sans doute de souvenirs remémorés et déformés, voire même d'hallucinations.

\subsection{Le thème de "I'homme accroupi »}

Outre ces brouillages des repères temporels, on remarque qu'un thème récurrent parcourt en filigrane les tableaux du Ch. 5. Il s'agit du thème de l'homme accroupi sur le pont, qui est déjà présent aux Ch. 2 et 4 et que l'on retrouve ensuite au Ch. 8.

$\mathrm{Au} \mathrm{Ch} .2$, le thème de l'homme accroupi apparaît dans trois passages non contigus :

(8) Sur le pont de rondins, qui franchit la rivière [...], il y a un homme accroupi. C'est un indigène, vêtu d'un pantalon bleu et d'un tricot de corps, sans couleur, qui laisse nues les épaules. Il est penché vers la surface liquide, comme s'il cherchait à voir quelque chose dans le fond, ce qui n'est guère possible, la transparence n'étant jamais suffisante malgré la hauteur d'eau très réduite. (Ch. $2, \S 16, \mathrm{p} .37$ )

(9) L'homme se tient toujours immobile, penché vers l'eau boueuse, sur le pont en rondins recouverts de terre. Il n'a pas bougé d'une ligne : accroupi, la tête baissée, les avant-bras s'appuyant sur les cuisses, les deux mains pendant entre les genoux écartés. (Ch. 2, § 24, p. 40)

(10) Sur le pont de rondins l'indigène accroupi a disparu. Il n'y a personne de visible aux alentours. Aucune équipe n'a affaire dans ce secteur, pour le moment. (Ch. 2, § 42, p. 42-43)

Ces trois passages correspondent, de la part de l'observateur, d'abord à une prise de conscience de l'existence d'un individu sur le pont (il y a un homme accroupi. C'est un indigène), puis à une nouvelle évocation du tableau, d'où la reprise du référent (l'homme) décrit dans la même position (toujours) et enfin à l'absence de l'individu, d'où l'effacement $\mathrm{du}$ tableau (l'indigène accroupi a disparu). Contrairement aux tableaux du Ch. 5, il n'est pas question ici de travaux sur le pont, pas même de préparatifs de réparation : il est précisé en (9) que les rondins sont recouverts de terre et en (10) qu'aucune équipe n'a affaire dans ce 
secteur, pour le moment. S'agirait-il de l'évocation d'une période encore antérieure, avant même qu'il soit question de réparer le pont? Ou bien cela remettrait-il en question la réalité des travaux de réparation si minutieusement décrits au Ch. 5 ?

$\mathrm{Au}$ Ch. 4 se retrouve une variante très proche de (8), avec toutefois quelques variations (trois ajouts : tout au fond de la vallée, petite rivière, tourné vers l'amont ; substitution de dans l'eau boueuse à dans le fond; suppression du commentaire ce qui n'est guère possible ... réduite ) :

(11) Tout au fond de la vallée, sur le pont de rondins qui franchit la petite rivière, il y a un homme accroupi, tourné vers l'amont. C'est un indigène, vêtu d'un pantalon bleu et d'un tricot de corps, sans couleur, qui laisse nues les épaules. Il est penché vers la surface liquide, comme s'il cherchait à voir quelque chose dans l'eau boueuse. (Ch. 4, § 1, p. 79-80)

Sans doute s'agit-il du retour (flash-back) au tableau initial de l'homme accroupi qui, pour quelque raison, a dû frapper l'esprit de l'observateur?

$\mathrm{Au}$ Ch. 5 (on l'a vu plus haut), la position accroupie est d'abord attribuée en (2) à tous les ouvriers, puis en (5) à un seul d'entre eux. En (2), la description précise de la position reprend celle de (9) avec deux petites variantes (suppression de la tête baissée et substitution de appuyés à s'appuyant) :

(2) Les ouvriers du pont sont au nombre de cinq, comme les troncs de rechange. Ils sont en ce moment tous accroupis dans la même position : les avant-bras appuyés sur les cuisses, les deux mains pendant entre les genoux écartés. (Ch. 5, § 13, p. 103-104)

En (5) l'ouvrier accroupi, comme l'indigène de (11), est tourné vers l'amont et regarde l'eau :

(5) La limite aval de ce trapèze est soulignée par la présence du chemin d'accès qui aboutit au petit pont sur le ruisseau. Les cinq hommes y sont maintenant ordonnés en quinconce, deux sur chaque berge et un au milieu, accroupi, tourné vers l'amont, regardant l'eau boueuse qui arrive dans sa direction entre deux parois de terre verticales, plus ou moins effondrées çà et là. (Ch. $5, \S 54$, p. 118)

Enfin, au Ch. 8 (en plein délire du mari), ce même thème de l'homme accroupi revient, juste après un paragraphe où le mari revoit en pensée A... dans la chambre regardant par une des fentes du volet et suit mentalement la direction du regard de celle-ci. La position accroupie de l'individu (l'homme) y est décrite dans des termes identiques à ceux de (9) modulo la substitution de est à se tient :

(12) L'homme est toujours immobile, penché vers l'eau boueuse, sur le pont en rondins recouverts de terre. Il n'a pas bougé d'une ligne : accroupi, la tête baissée, les avant-bras s'appuyant sur les cuisses, les deux mains pendant entre les genoux écartés. Il a l'air de guetter quelque chose, au fond de la petite rivière - une bête, un reflet, un objet perdu. (Ch. 8, § 2, p. 182-183)

Mais (12) comporte une modification intéressante par rapport à (9) : la notation comme s'il cherchait à voir quelque chose dans le fond est devenue il a l'air de guetter quelque chose au fond [...] - une bête, un reflet, un objet perdu. La reformulation n'est pas sans évoquer l'attitude du mari lui-même, qui cherche à voir, en quête d'un indice: lui aussi est obsédé par une bête (la scutigère liée au thème de la tache), par un reflet (celui du cadre en nacre de la photographie de sa femme qu'il contemple en son absence) et par un objet qui lui échappe (la lettre). Le thème de l'homme accroupi renvoie donc métaphoriquement au thème de l'impureté, c'est-à-dire au motif de la tache: tout comme l'homme accroupi, le mari n'arrive pas à voir clair à travers la boue, faute de transparence. Or le caractère impur, non- 
transparent des choses n'existe qu'aux yeux d'un observateur humain (l'indigène/le mari), comme le souligne, dans les dernières pages du roman, le passage suivant :

(13) Tout en bas, au fond de la vallée, [...], l'eau de la petite rivière montre une surface plissée, qui témoigne de la rapidité du courant. [...]. Le flot s’écoule, mais la surface reste comme figée [...].

L'éclat, de même, en est fixe et donne à la nappe liquide un aspect plus transparent. Mais il n'y a personne pour en juger sur place, depuis le pont par exemple. [...].

(Ch. 9, § 9-10, p. 213)

En définitive, la scène du pont se présente donc comme un kaléidoscope d'images partiellement identiques, partiellement différentes (voire contradictoires), où semblent se mêler indissociablement perceptions, souvenirs et fantasmes. De plus, l'entrelacement avec le thème de l'homme accroupi indique que cette scène est, pour le mari, l'occasion de projeter ses propres obsessions sur les images qu'il perçoit ou qu'il convoque à cette occasion. Pour un lecteur soucieux de retrouver un « fil » temporel linéaire, la cohérence du récit se trouve affectée par ce brouillage des repères (spatio-)temporels. Mais, comme nous le verrons au $\S 3$, un tel brouillage, volontaire de la part de l'auteur, est précisément destiné à bloquer le fil du temps en instaurant une sorte de présent de conscience des images qui se superposent, et ce n'est pas dans la recherche d'une chronologie référentialiste qu'il convient de chercher la clé de lecture.

\section{La scène de l'apéritif}

La seconde scène sur laquelle nous nous pencherons est celle de l'apéritif pris sur la terrasse de la maison par les trois protagonistes: Franck, A... et le mari ${ }^{4}$. Cette scène concerne directement des faits et gestes de A... et de Franck, épiés par le mari. Elle occupe donc une place plus importante que la précédente dans l'économie du roman : elle figure dans six des neufs chapitres (aux Ch. 1, 2, 4, 5, 8 et 9). Comme nous allons le voir, cette scène, si souvent évoquée à travers un jeu incessant de répétitions et de déformations, est particulièrement problématique à reconstruire de façon stable: les multiples variantes construisent une sorte d'effet stroboscopique qui contribue à désorienter le lecteur.

Contrairement à la scène du pont, la scène de l'apéritif est difficile à délimiter au sein du texte : les passages sont entrecoupés de digressions et leurs frontières mutuelles sont floues. Voici, très schématiquement, comment se présentent les chapitres mentionnant la scène de l'apéritif :

30 - Au Ch. 1, un passage ( $\$ 22-27$, p. 17-20) relate un apéritif pris le soir, avant un dîner où Franck et sa femme Christiane étaient invités, mais cette dernière ne venant pas, A... fait retirer son couvert.

31 - Au Ch. 2, un premier passage (\$35-72, p. 35-52) parle d’un apéritif avant un déjeuner (Franck étant venu impromptu, A... fait ajouter son couvert). Suit le récit d'un dîner à trois dans la salle à manger, l'évocation d'une camionnette bâchée et d'A... ramenée en voiture par Franck (§ 73-94). Puis un passage (\$ 95-97, p. 58-59) parlant d'un apéritif $d u$ soir, avant un dîner à trois où il est question d'un futur voyage en voiture jusqu'à la ville : Franck propose d'y emmener A...

32 - Au Ch. 4, un premier passage (commençant au $\S 4$, p. 81, mais dont la frontière finale n'est pas nette) a trait à un apéritif où Franck et A... planifient les horaires de leur futur voyage puis parlent d'un roman qu'ils ont lu. Alors que le tableau de ce même apéritif semble se poursuivre, le lecteur découvre brusquement (\$21, p.84) que les deux 
personnages sont à présent en train de commenter l'incident mécanique qui s'est produit lors de leur voyage ( $\$ 21-38$, p. 84-87)!; Franck, qui a déposé A... en passant, s’en va (§ 39-40, p. 87-88).

- Au Ch. 5, un passage ( $\$ 17-37$, p. 105-110) semble à première vue relater un unique apéritif ; mais diverses contradictions incitent un lecteur attentif à conclure qu'il s'agit de tableaux distincts.

- Au Ch. 8 (le plus touffu à cet égard), un premier passage (\$19-61, p. 189-197) concerne un apéritif où le futur voyage est évoqué pour la semaine suivante. Puis, après la mention d'un déjeuner à trois sans Christiane (où il est fait allusion à l'incident du voyage passé !), un deuxième passage ( $\$ 75-79$, p. 200-201) présente la fin d'un apéritif où Franck, de passage, part rapidement. Sont ensuite évoqués un déjeuner à deux (le mari et A..., qui reprend ensuite sur la terrasse sa « lecture interrompue par l'arrivée de Franck »), puis le bruit d'une voiture et le retour du voyage d'A... et de Franck le matin. Un troisième passage (\$ 92, p. 205) mentionne un bref apéritif. Enfin, un quatrième passage (\$ 94-100, p. 205-208) relate un apéritifle soir avant un dîner (où Christiane, qui était attendue, ne vient pas : A... fait enlever son couvert) pendant lequel est évoqué le projet de voyage en ville!

- Au Ch. 9, A... revient d'une visite à Christiane, reconduite par Franck. Un passage (§ 13-17, p. 214-217) relate un apéritif du soir où Franck prend rapidement congé.

Le lecteur se perd donc en conjectures : apéritif(s) de midi ou du soir? apéritif(s) sur invitation ou impromptu(s)? apéritif(s) avant le voyage à la ville ou au retour? Le nombre et la longueur des passages concernés ne nous permettent pas de procéder à une mise en parallèle exhaustive. Nous nous centrerons donc sur la façon dont les différentes variantes décrivent les fauteuils, les boissons, le projet de voyage d'A... et de Franck, et le roman qu'ils lisent ${ }^{5}$.

\subsection{Les fauteuils}

Dans les différentes versions de la scène de l'apéritif, les indications qui se retrouvent de façon stable concernent les objets (la description des fauteuils) et l'espace (leur disposition sur la terrasse et la place de chacun des protagonistes).

38 Trois des fauteuils sont des fauteuils très simples, en bois et sangles de cuir, exécutés sur les indications de A... par un artisan indigène (Ch. 1, § 22, p. 18)/des fauteuils de fabrication locale (Ch. 2, § 35, p. 44)/des fauteuils tendus de cuir (Ch. 2, §95, p. 58), tandis qu'un autre fauteuil est un siège pliant fait de toile tendue sur des tiges métalliques (Ch. 1, § 26, p. 20).

39 Deux des fauteuils indigènes se trouvent côte à côte, contre le mur de la maison [...] sous la fenêtre du bureau (Ch. 1, § 25, p. 19)/devant les fenêtres du bureau (Ch. 2, § 35, p. 44)/sous la fenêtre du bureau (Ch. 8, § 19 p. 189), tandis que l'autre fauteuil (ou les deux autres fauteuils, selon le cas), est placé en retrait.

40 Chacun des protagonistes a sa place attitrée : A... et Franck dans les deux fauteuils côte à côte et (on le comprend implicitement) le mari dans le fauteuil le plus éloigné. Cette disposition a été voulue par A... (pour des raisons de vue depuis la terrasse, est-il dit).

41 Cette stabilité des repères spatiaux donne, à première vue, une impression de cohérence et d'apparente « objectivité ». On constate pourtant que nombre de variantes comportent des notations subjectives attribuables au mari. Certaines expriment sa contrariété face à la disposition des fauteuils voulue par A..., qui l'empêche d'épier facilement celle-ci (c'est nous qui soulignons dans tous les exemples qui suivent): 
(14) C'est elle-même [= A...] qui a disposé les fauteuils, ce soir, quand elle les a fait apporter sur la terrasse. Celui qu'elle a désigné à Franck et le sien se trouvent côte à côte, contre le mur de la maison - le dos vers ce mur, évidemment - sous la fenêtre $\mathrm{du}$ bureau. Elle a ainsi le fauteuil de Franck à sa gauche, et sur sa droite - mais plus en avant - la petite table où sont les bouteilles. Les deux autres fauteuils sont placés de l'autre côté de cette table, davantage encore vers la droite, de manière à ne pas intercepter la vue entre les deux premiers et la balustrade de la terrasse. Pour la même raison de "vue ", ces deux derniers fauteuils ne sont pas tournés vers le reste du groupe : ils ont été mis de biais, orientés obliquement vers la balustrade à jours et l'amont de la vallée. Cette disposition oblige les personnes qui s'y trouvent assises à de fortes rotations de tête vers la gauche, si elles veulent apercevoir A... - surtout en ce qui concerne le quatrième fauteuil, le plus éloigné. [...]. (Ch. 1, § 25-26, p. 19-20)

D'autres insistent sur son constant agacement face au sans-gêne de Franck, qui se comporte en habitué de la maison :

(15) Sur la terrasse, Franck se laisse tomber dans un des fauteuils bas et prononce son exclamation - désormais coutumière - au sujet de leur confort. [...]. (Ch. 1, § 22, p. 17-18)

(16) Sur la terrasse, devant les fenêtres du bureau, Franck est assis à sa place habituelle, dans un des fauteuils de fabrication locale. Seuls ces trois-là ont été sortis ce matin. Ils sont disposés comme à l'ordinaire : les deux premiers rangés côte à côte sous la fenêtre, le troisième un peu à l'écart, de l'autre côté de la table basse. (Ch. 2, § 35, p. 44)

(17) Celui-ci [= Franck] est encore là pour le dîner, affable et souriant. Il se laisse tomber dans un des fauteuils tendus de cuir, sans que personne le lui ait désigné, et prononce son exclamation coutumière au sujet de leur confort :

«Ce qu'on est bien là-dedans!»

Sa chemise blanche fait une tache plus pâle dans la nuit, contre le mur de la maison. (Ch. 2, § 95-97, p. 58)

(18) De l'autre côté de la porte du couloir, sous la fenêtre symétrique, une de celles du bureau, Franck est assis dans son fauteuil. (Ch. 5, § 17, p. 105)

(19) À la même distance, mais dans une direction perpendiculaire, Franck et A... sont en train de boire l'apéritif, renversés en arrière dans leurs deux fauteuils coutumiers, sous la fenêtre du bureau. «Ce qu'on est bien là-dedans ! ». [...]. (Ch. 8, $\S 19$, p. 189)

(20) Celui-ci [= Franck] est assis dans son fauteuil, sous la première fenêtre du bureau. [...]. (Chapitre 9, § 13, p. 214)

43 Par ailleurs, certaines distorsions factuelles sont repérables: Franck prend-il le siège qu'A... lui désigne (14) ou bien s'assied-il spontanément dans son fauteuil (17) ? Les altérations peuvent aller jusqu'à la contradiction. Ainsi, s'agissant du siège pliant, qui est demeuré vide ou au contraire que le boy a rajouté:

(21) Le troisième [siège], qui est un siège pliant fait de toile tendue sur des tiges métalliques, occupe - lui - une position nettement en retrait, entre le quatrième et la table. Mais c'est celui-là, moins confortable, qui est demeuré vide. (Ch. 1, § 26, p. 20)

(22) Le soir la [= A...] trouve dans la même posture, dans le même fauteuil, devant le même lézard en pierre grise. La seule différence est que le boy a rajouté le quatrième siège, celui qui est moins confortable, fait de toile tendue sur des tiges métalliques. [...]. (Ch. 8, § 94, p. 205)

Le tableau (22) est une évocation d'A... sur la terrasse, dans l'esprit du mari resté seul lors de l'attente du retour des deux voyageurs : il la revoit, l'imagine et fantasme, obsédé sans doute par le scénario inverse du siège/du couvert enlevé/rajouté. 


\subsection{Les boissons} variantes comportent à la fois des reformulations presque verbatim et des divergences notoires. Le schéma de base qui sous-tend toutes les versions est le suivant : A... apporte les boissons (eau gazeuse et cognac) sur un plateau; elle verse le mélange dans les trois verres et les apporte, dans l'ordre, à Franck puis au mari (ceci restant implicite) avant de s'asseoir avec son propre verre. Deux séquences textuelles figurant dans le premier récit (23) de la scène de l'apéritif se retrouvent pratiquement à l'identique dans l'une ou l'autre des variantes ultérieures (c'est nous qui les soulignons dans tous les exemples qui suivent) :

(23) [...]. Elle [= A...] se penche vers Franck pour lui tendre son verre.

Bien qu'il fasse tout à fait nuit maintenant, elle a demandé de ne pas apporter les lampes, qui - dit-elle - attirent les moustiques. Les verres sont emplis, presque jusqu'au bord, d'un mélange de cognac et d'eau gazeuse où flotte un petit cube de glace. Pour ne pas risquer d'en renverser le contenu par un faux mouvement, dans l'obscurité complète, elle s'est approchée le plus possible du fauteuil où est assis Franck, tenant avec précaution dans la main droite le verre qu'elle lui destine. Elle s'appuie de l'autre main au bras du fauteuil et se penche vers lui, si près que leurs têtes sont l'une contre l'autre. Il murmure quelques mots : un remerciement, sans doute.

Elle se redresse d'un mouvement souple, s'empare du troisième verre - qu'elle ne craint pas de renverser, car il est beaucoup moins plein - et va s'asseoir à côté de Franck, [...]. (Ch. 1, § 22-24, p. 18-19)

(24) A... est en train de verser l'eau minérale dans les trois verres, alignés sur la table basse. Elle distribue les deux premiers, puis, tenant le troisième en main, va s'asseoir dans le fauteuil vide, à côté de Franck. Celui-ci a déjà commencé à boire. (Ch. 2, § 43, p. 46)

(25) Pour ne pas risquer d'en renverser le contenu par un faux mouvement, dans l'obscurité complète, A... s'est approchée le plus possible du fauteuil où est assis Franck, tenant avec précaution dans la main droite le verre qu'elle lui destine. Elle s'appuie de l'autre main au bras du fauteuil et se penche vers lui, si près que leurs têtes sont l'une contre l'autre. Il murmure quelques mots: sans doute un remerciement. Mais les paroles se perdent dans le vacarme assourdissant des criquets qui monte de toutes parts. (Ch. 2, \& 98, p. 58-59)

(26) A..., qui est allée chercher elle-même les boissons, dépose le plateau chargé sur la table basse. Elle débouche le cognac et en verse dans les trois verres alignés. Elle les emplit ensuite avec l'eau gazeuse. Ayant distribué les deux premiers, elle va s'asseoir à son tour dans le fauteuil vide, tenant le troisième en main. (Ch. 5, $\S 18$, p. 105)

Mais les différents récits divergent sur un point, qui a trait aux glaçons destinés à rafraîchir les boissons. Dans le premier récit du Ch. 1, les glaçons sont présents dans les verres :

(23) [...]. Les verres sont emplis, presque jusqu'au bord, d'un mélange de cognac et d'eau gazeuse où flotte un petit cube de glace. [...]. (Ch. 1, § 23, p. 18)

Dans la (ou les ?) version(s) du Ch. 2, les glaçons sont au contraire absents. Le passage (24) est en effet suivi d'une séquence où $\mathrm{A}$... appelle le boy pour qu'il les apporte et la précision suivante est donnée : Du reste, une seule bouteille vient du réfrigérateur : l'eau minérale [...]. Le cognac, lui, reste toujours dans le buffet. A..., qui chaque jour apporte le seau à glace en même temps que les verres, ne l'a pas fait aujourd'hui (Ch. 2, § 52-53 p. 48). 

service :

(27) A... est elle-même allée chercher les boissons, eau gazeuse et cognac. Elle dépose sur la table un plateau chargé portant les deux bouteilles et trois grands verres. Ayant débouché le cognac, elle se tourne vers Franck et le regarde, tandis qu'elle commence à le servir. [...]. (Ch. 2, § 36, p. 44-45)

De même, au Ch. 5, les glaçons sont manquants. Juste à la suite du passage (26) cité plus haut, est à nouveau mentionné l'appel au boy pour qu'il apporte les glaçons. Mais loin de constituer une simple indication factuelle, la mention prend ici de fortes connotations subjectives qui dévoilent le point de vue du jaloux sur des faits qu'il se remémore : C'est alors qu'elle $[=A$....] demande si les habituels cubes de glace seront nécessaires, prétextant que ces bouteilles sortent du réfrigérateur, une seule des deux pourtant s'étant couverte de buée au contact de l'air (Ch. 5, § 19, p. 106).

A contrario, au Ch. 4, les glaçons sont présents (comme au tableau du Ch. 1) :

(28) A... vient d'apporter les verres, les deux bouteilles et le seau à glace. Elle commence à servir : le cognac dans les trois verres, puis l'eau minérale, enfin trois cubes de glace transparente qui emprisonnent en leur cœur un faisceau d'aiguilles argentées. (Ch. 4, § 4, p. 81)

51 Cette contradiction à propos de ce qui pourrait apparaître comme un détail mineur trahit au contraire l'importance, pour l'observateur, du motif des glaçons : le mari y voit en effet un stratagème de sa femme pour l'éloigner un instant (on comprend qu'il se rend à la cuisine pour parler au boy), au moment où elle aperçoit la lettre qui dépasse de la poche de Franck. Et en (28), la description des glaçons (trois cubes de glace transparente qui emprisonnent en leur coeur un faisceau d'aiguilles argentées) peut être lue comme le symbole de la douleur et de l'angoisse (faisceau d'aiguilles) qui enserre le cour du mari, face à l'énigme des yeux (glace transparente) de sa femme (dont il est dit à plusieurs reprises dans le roman qu'ils ne cillent jamais).

Le motif du cube de glace se retrouve encore dans d'autres tableaux (également contradictoires, y compris au sein d'un même chapitre) de la scène de l'apéritif :

(29) Ils boivent à petites gorgées. Dans les trois verres, les morceaux de glace ont maintenant tout à fait disparu. Franck examine ce qui reste de liquide doré, au fond du sien. [...]. (Ch. 4, § 20, p. 83-84)

(30) Franck se lève de son fauteuil, avec une vigueur soudaine, et pose sur la table basse le verre qu'il vient de finir d'un trait. Il n'y a plus trace du cube de glace dans le fond. [...]. (Ch. 5, § 29, p. 108)

(31) Au fond du verre qu'il a déposé sur la table en partant, achève de fondre un petit morceau de glace, arrondi d'un côté, présentant de l'autre une arête en biseau. [...]. (Ch. 5, § 32, p. 109)

\subsection{Le voyage à la ville et le roman}

53 Dans les récits de l'apéritif, le voyage à la ville de A... en compagnie de Franck est évoqué à trois reprises comme un projet. La première évocation figure au Ch. 4 (juste à la suite de (28) décrivant les cubes de glace), sous forme d'un dialogue :

(32) « Nous partirons de bonne heure, dit Franck.

- C'est-à-dire?

- Six heures, si vous voulez bien.

- Oh ! là là...

- Ça vous fait peur? 
- Mais non. » Elle rit. Puis, après un silence : «Au contraire, c'est très amusant. »

Ils boivent à petites gorgées.

«Si tout va bien, dit Franck, nous pourrions être en ville vers dix heures et avoir

déjà pas mal de temps avant le déjeuner.

- Bien sûr, je préfère aussi ", dit A...

- Ils boivent à petites gorgées. (Ch. 4, § 5-14, p. 81)

54

La seconde se trouve au Ch. 8 , sous la forme d'un court récit, très factuel :

(33) [...]. Ils parlent, à bâtons rompus, du voyage en ville qu'ils ont l'intention de faire ensemble, dans le courant de la semaine suivante, elle pour diverses courses, lui pour se renseigner au sujet du nouveau camion qu'il a projeté d'acquérir. (Ch. 8 , $\S 20$, p. 190)

La troisième, située un peu plus loin au même Ch. 8 , se présente comme une reformulation de (32), suivie du rajout d'un dernier tour de parole de Franck:

(34) Franck reprend :

« Nous partirons de bonne heure.»

A... réclame des précisions. Franck les donne et s'inquiète de savoir si c'est trop tôt pour sa passagère.

"Au contraire, dit-elle, c'est très amusant. »

Ils boivent à petites gorgées.

«Si tout va bien, dit Franck, nous pourrions être en ville vers dix heures et avoir déjà pas mal de temps avant le déjeuner.

- Bien sûr, je préfère aussi, répond A... dont la mine est redevenue sérieuse.

- Ensuite je n'aurai pas trop de tout l'après-midi pour terminer mes visites aux divers agents ; et prendre aussi l'avis du garagiste chez qui je vais toujours, Robin, vous savez, sur le front de mer. Nous rentrerons aussitôt après dîner." (Ch. 8, $\S 53-60$, p. 196)

Mais la variante bifurque, car ce dernier propos de Franck donne lieu au commentaire suivant - qui conduit à lire (34) comme l'évocation après coup par le mari de la planification du voyage, objet de ses soupçons :

(35) Les précisions qu'il fournit sur son emploi du temps futur, pour cette journée en ville, seraient plus naturelles si elles venaient satisfaire quelque demande d'un interlocuteur; personne n'a pourtant manifesté le moindre intérêt, aujourd'hui, concernant l'achat de son camion neuf. Et pour un peu il établirait à haute voix - à très haute voix - le détail de ses déplacements et de ses entrevues, mètre par mètre, minute par minute, en appuyant à chaque fois sur la nécessité de sa conduite. A..., en revanche, ne fait pas le plus petit commentaire quant à ses propres courses, dont la durée globale sera la même, cependant (Ch. 8, § 61, p. 196-197)

57 Dans les passages (32) et (34) ci-dessus, figure par trois fois la phrase Ils boivent à petites gorgées (également présente en (29)). Cette notation contraste avec une autre expression: le verre qu'il [= Franck] vient de finir d'un trait, présente en (30) et figurant aussi à l'avantdernier chapitre : Franck, venu juste en passant, déclare qu'il ne veut pas s'attarder davantage. Il se lève en effet de son fauteuil et pose sur la table basse le verre qu'il vient de finir d'un trait. (Ch. 8, § 78, p. 200). S'agirait-il, là encore, d'une métaphore de l'acte sexuel ?

De son côté, le roman - lecture commune de Franck et de A..., qui alimente leurs conversations - est mentionné dans les trois passages suivants, qui divergent quant à l'état d'avancement de la lecture (nouveau brouillage des repères temporels) :

(36) Ensuite ils parlent d'autre chose. Ils ont achevé maintenant l'un comme l'autre la lecture de ce livre qui les occupe depuis quelque temps; [...]. (Ch. 4, § 15, p. 82)

(37) Cinq ou six phrases sont alors échangées sur les doses respectives de quinine nécessaires dans les différentes zones tropicales, [...]. Puis Franck revient aux effets 
fâcheux que produit la quinine sur l'héroïne du roman africain que A... est en train de lire. [...]. (Ch.8, § 40, p. 193)

(38) La lumière décroît rapidement. A..., qui ne voit plus assez clair pour continuer sa lecture, ferme son roman et le repose sur la petite table, à côté d'elle [...]. Pour marquer la page, le rebord de la jaquette vernie protégeant la couverture a été repliée à l'intérieur du livre, au premier quart environ de son épaisseur. (Ch. 8, $\S 95$, p. 206)

La scène de l'apéritif se présente ainsi comme une sorte de puzzle dont les morceaux ne s'emboîteraient pas: une série de tableaux partiellement identiques et partiellement divergents (voire contradictoires), constitués de perceptions fugitives, de notations brèves, qui reviennent comme dans le désordre, rendant impossible une reconstitution stabilisée des faits. Incapable de savoir quand commence ou se termine un tableau, et donc de déterminer le nombre d'apéritifs distincts décrits, le lecteur renonce finalement à chercher au sein des variantes «la vraie » version de référence, celle qui aurait pu donner lieu aux nombreuses altérations et bifurcations. Comme nous allons le voir à présent, ce jeu sur les répétitions/déformations constitue l'un des ressorts principaux de la stratégie d'écriture de l'auteur.

\section{Une stratégie d'écriture}

Les divers mécanismes d'auto-reformulation à l'œuvre dans les variantes des deux scènes que nous venons d'étudier participent d'une stratégie d'écriture très consciente de la part d'A. Robbe-Grillet. Celui-ci en a d'ailleurs lui-même détaillé les principes et les motivations dans plusieurs écrits (voir notamment A. Robbe-Grillet - en bref RG - 1961 et 2013 [1963]). Par ailleurs, une lecture attentive de La Jalousie permet de détecter certaines clés (concernant les effets volontairement visés par ce type d'écriture) qui ont été subrepticement glissées dans le roman même par l'auteur.

\subsection{Le brouillage des repères (spatio-) temporels et le statut des images}

61 Le contenu de chacune des scènes est constitué d'un kaléidoscope d'images, sortes d'instantanés présents à la conscience de l'observateur, ayant toutes le même statut de réalité pour lui. Ces images, on l'a vu, sont relativement stables quant à la description (parfois précise jusqu'à la maniaquerie ${ }^{6}$ ) des objets et des lieux (qui sont des éléments extérieurs à l'observateur) - encore que cette stabilité se mette à vaciller aux moments de grande angoisse et de délire. Mais elles sont totalement instables quant aux repères dans le temps (éléments liés à la conscience et à la subjectivité), précisément parce qu'elles mêlent perceptions immédiates, souvenirs, rêves et fantasmes.

Le brouillage des repères (spatio-)temporels témoigne, chez A. Robbe-Grillet, de sa «tentative de construire un espace et un temps purement mentaux »:

ceux du rêve peut-être, ou de la mémoire, ceux de toute vie affective - sans trop s'occuper des enchaînements traditionnels de causalité, ni d'une chronologie absolue de l'anecdote. (RG, 1961, pp. 9-10)

C'est pourquoi il s'insurge contre une lecture « référentialiste » de ses œuvres :

Pourquoi chercher à reconstituer le temps des horloges dans un récit qui ne s'inquiète que de temps humain? N'est-il pas plus sage de penser à notre propre mémoire, qui n'est jamais chronologique? (RG, 2013, p. 150) 
64 les images construites par la perception immédiate:

Une imagination, si elle est assez vive, est toujours au présent. Les souvenirs que l'on « revoit ", les régions lointaines, les rencontres à venir, ou même les épisodes passés que chacun arrange dans sa tête en modifiant le cours tout à loisir, il y a là comme un film intérieur qui se déroule continuellement en nous-mêmes, dès que nous cessons de prêter attention à ce qui se passe autour de nous. Mais, à d'autres moments, nous enregistrons au contraire, par tous nos sens, ce monde extérieur qui se trouve bel et bien sous nos yeux. Ainsi le film total de notre esprit admet à la fois tour à tour et au même titre les fragments réels proposés par la vue et l'ouïe, et des fragments passés, ou lointains, ou futurs, ou totalement fantasmagoriques. (RG, 1961, p. 16).

Les images peuvent être déformées parce qu'elles sont affectivement chargées pour l'observateur humain :

Non seulement c'est un homme qui, dans mes romans par exemple, décrit toute chose, mais c'est le moins neutre, le moins impartial des hommes: engagé au contraire toujours dans une aventure passionnelle des plus obsédantes, au point de déformer souvent sa vision et de produire chez lui des imaginations proches $d u$ délire. (RG, 2013, p. 149)

\subsection{Répétitions, variantes et bifurcations}

66 À la lecture, les répétitions incessantes de certaines images - parfois légèrement déformées, parfois totalement altérées - créent une impression d'étrangeté. Toujours désarçonné dans son (vain) effort pour reconstruire la «vraie» réalité logique et chronologique, le lecteur navigue d'une image à l'autre, ballotté entre des tableaux fragmentaires qui ne se raccordent pas, dérouté par la survenue abrupte de motifs obsessionnels qui font dévier le récit et semblent l'empêcher de progresser.

67 Cette perplexité du récepteur se trouve évoquée à deux reprises par A. Robbe-Grillet luimême dans La Jalousie, à propos d'une mélodie chantée par un indigène ${ }^{7}$ - tout aussi étrange à l'oreille de l'auditeur occidental que l'est le roman pour un lecteur habitué aux récits linéaires (c'est nous qui soulignons dans tous les exemples qui suivent) :

(39) A cause du caractère particulier de ce genre de mélodie, il est difficile de déterminer si le chant s'est interrompu pour une raison fortuite [...] ou bien si l'air trouvait là sa fin naturelle.

De même, lorsqu'il recommence, c'est aussi subtil, aussi abrupt, sur des notes qui ne semblent guère constituer un début, ni une reprise.

A d'autres endroits, en revanche, quelque chose semble en train de se terminer; tout l'indique : une retombée progressive, le calme retrouvé, le sentiment que plus rien ne reste à dire; mais après la note qui devait être la dernière en vient une suivante, sans la moindre solution de continuité, avec la même aisance, puis une autre, et d'autres à la suite, et l'auditeur se croit transporté en plein cœur du poème... quand, là, tout s'arrête, sans avoir prévenu. (Ch. 5, § 4-6, p. 100-101)

(40) Le poème ressemble si peu, par moment, à ce qu'il est convenu d'appeler une chanson, une complainte, un refrain, que l'auditeur occidental est en droit de se demander s'il ne s'agit pas de tout autre chose. Les sons, en dépit d'évidentes reprises, ne semblent liés par aucune loi musicale. Il n'y a pas d'air, en somme, pas de mélodie, pas de rythme. On dirait que l'homme se contente d'émettre des lambeaux sans suite pour accompagner son travail. [...]. (Ch. 8, § 47, p. 194-195)

Ce qu'A. Robbe-Grillet attend du lecteur, ce n'est pas qu'il se livre à une lecture naïve en quête d'une histoire et de personnages auxquels s'identifier ${ }^{8}$, mais qu'il se laisse porter 
par les images et trouve un autre niveau de cohérence, en s'intéressant à l'écriture ellemême et à ses ressorts profonds. En témoigne le passage suivant où, évoquant les discussions entre A... et Franck à propos du roman qu'ils lisent, le narrateur attribue à ces derniers le type de lecture qu'il incrimine :

(41) Jamais ils n'ont émis au sujet du roman le moindre jugement de valeur, parlant au contraire des lieux, des événements, des personnages, comme s'il se fût agi de choses réelles : un endroit dont ils se souviendraient (situé d'ailleurs en Afrique), des gens qu'ils y auraient connus, ou dont on leur aurait raconté l'histoire. Les discussions, entre eux, se sont toujours gardées de mettre en cause la vraisemblance, la cohérence, ni aucune qualité du récit. En revanche il leur arrive souvent de reprocher aux héros eux-mêmes certains actes, ou certains traits de caractère, comme ils le feraient pour des amis communs. (Ch. 4, § 16, p. 82)

La cohérence du récit est en effet à rechercher dans la réalité qui est celle des images pour l'observateur : à savoir leur inscription dans un présent (maintenant) intemporel, celui des divers états de conscience - chaque déformation, si minime soit-elle, constituant précisément l'indice d'un changement d'état de conscience, tout comme elle signale une modification dans le cours du chant :

(42) Sans doute est-ce toujours le même poème qui se continue. Si parfois les thèmes s'estompent, c'est pour revenir un peu plus tard, affermis, à peu de choses près identiques. Cependant ces répétitions, ces infimes variantes, ces coupures, ces retours en arrière, peuvent donner lieu à des modifications - bien qu'à peine sensibles - entraînant à la longue fort loin du point de départ. (Ch. $5, \S 8$, p. 101)

Cette stratégie d'écriture n'est toutefois pas exempte d'une certaine duplicité. Car, comme A... et Franck à propos du roman qu'ils lisent, peut-être A. Robbe-Grillet se laisse$\mathrm{t}$-il lui aussi prendre au jeu des bifurcations ${ }^{9}$ et griser par la prolifération des variantes :

(43) Ils déplorent aussi quelquefois les hasards de l'intrigue, disant que «ce n'est pas de chance ", et ils construisent alors un autre déroulement probable à partir d'une nouvelle hypothèse, «si ça n'était pas arrivé ». D'autres bifurcations possibles se présentent, en cours de route, qui conduisent toutes à des fins différentes. Les variantes sont très nombreuses; les variantes des variantes encore plus. Ils semblent même les multiplier à plaisir, échangeant des sourires, s'excitant au jeu, sans doute un peu grisés par cette prolifération (Ch. $4, \S 17$, p. 83)

71 Car à vouloir ainsi «brouiller les pistes » et mettre en échec la recherche d'une stabilité référentielle objective, l'on peut se retrouver dans une impasse - c'est-à-dire dans l'impossibilité d'opter entre des interprétations contradictoires, à l'instar des deux protagonistes commentant le roman qu'ils lisent :

(44) Le personnage principal du livre est un fonctionnaire des douanes. Le personnage n'est pas un fonctionnaire, mais un employé supérieur d'une vieille compagnie commerciale. Les affaires de cette compagnie sont mauvaises, elles évoluent rapidement vers l'escroquerie. Les affaires de la compagnie sont très bonnes. Le personnage principal - apprend-on - est malhonnête. Il est honnête, il essaie de rétablir une situation compromise par son prédécesseur, mort dans un accident de voiture. Mais il n'a pas eu de prédécesseur, car la compagnie est de fondation toute récente; et ce n'était pas un accident. Il est d'ailleurs question d'un navire (un grand navire blanc) et non de voiture. (Ch. 9, § 16, p. 216)

N'est-ce pas face à une telle impasse que se trouve aussi le spectateur qui, sortant du cinéma, ne sait toujours pas si l'héroïne du film était présente ou non, l'année dernière à 
Marienbad? A. Robbe-Grillet, amateur de labyrinthes, se joue décidément de nous (et de lui-même) à travers l'inextricable réseau des répétitions, variantes et bifurcations.

\section{BIBLIOGRAPHIE}

LEBRAVE, J.-L. (2014). « Genèse d'une traduction : comment Elmar Tophoven a annoté La Jalousie d'Alain Robbe-Grillet ». Genesis 38, p. 35-56.

ROBBE-GRILLET, A. (1957). La Jalousie. Paris : Minuit.

- (1961). « Introduction ». In : Robbe-Grillet, A, L'année dernière à Marienbad, ciné-roman. Paris :

Minuit, p. 7-19.

- (1963) [2013]. Pour un Nouveau Roman. Paris : Minuit.

SARDA, L. \& LE GOFFIC, P. (2016). « Localisation et perception : l'observateur invisible

omniprésent ». In : Fuchs, C. (dir.), L'espace de La Jalousie : traduire, transférer, transposer. Caen :

Presses de l'université de Caen, p. 59-93.

\section{NOTES}

1. Il va sans dire qu'une telle récurrence de séquences reprises/déformées exige, de la part des traducteurs, une attention particulière. Il est intéressant, à cet égard, de savoir que E. Tophoven, responsable de la traduction allemande du roman (parue en 1959 aux éditions Hanser à Münich sous le titre Die Jalousie oder die Eifersucht), avait annoté son exemplaire de l'original français, en soulignant celles des reformulations qu'il repérait au fil du texte (Lebrave, 2014).

2. Pour une analyse détaillée de la scène de l'écrasement de la scutigère, voir L. Sarda \& P. Le Goffic (2016, § 2.3).

3. Bien que l'ouvrage ne comporte pas de numérotation explicite en chapitres, il apparaît néanmoins que le texte est divisé en 9 sections - le passage d'une section à l'autre étant simplement matérialisé, au fil du texte, par un espacement plus important entre les paragraphes, sans saut de page. La «Table » figurant à la fin du roman confirme ce découpage en 9 sections chacune d'entre elles étant désignée, non pas par un numéro, mais par les premiers mots de son début, et suivie du numéro de page correspondant.

4. La présence de ce dernier, observateur invisible omniprésent, n'est jamais mentionnée explicitement et ne se laisse inférer qu'à partir d'un certain nombre d'indices soigneusement disséminés au fil du texte, comme par exemple la mention d'un troisième verre.

5. D'autres points auraient évidemment mérité discussion, dont nous ne parlerons pas ici faute de place, comme par exemple la description des vêtements, des gestes et des attitudes des deux supposés amants.

6. Peut-être la formation initiale d'ingénieur agronome qui était celle de l'auteur explique-t-elle en partie la minutie et les précisions quasi-mathématiques de certaines notations spatiales.

7. Nous rejoignons ici L. Sarda et P. Le Goffic (2016, § 2.2.), qui relèvent eux aussi le passage (39) comme « une image de la construction même du texte où il serait vain de vouloir chercher une logique absolue ». 
8. Il n'aura de cesse de dénoncer cette « atmosphère anthropocentrique, vague mais baignant toutes choses, donnant à toute chose sa signification, c'est-à-dire l'investissant de l'intérieur par un réseau plus ou moins sournois de sentiments et de pensées » (2013, p. 58).

9. Jeu qui n'est pas sans rappeler les bifurcations du récit dans le film d'Alain Resnais Smoking, No Smoking.

\section{RÉSUMÉS}

La présente étude porte sur les mécanismes d'autoreformulation dans le roman La Jalousie d'A. Robbe-Grillet. Les deux premières sections sont consacrées aux multiples micro-variations observables dans les descriptions réitérées de deux scènes particulières (la réparation d'un pont au fond de la vallée et l'apéritif sur la terrasse) et aux effets, sur un lecteur soucieux de cohérence référentielle, des subtiles déformations à l'œuvre. La troisième section s'attache à la stratégie d'écriture revendiquée par l'auteur, qui s'inscrit dans le courant du Nouveau Roman; il est montré que le jeu incessant des altérations/bifurcations, qui brouille les repères spatiotemporels et désoriente ainsi le lecteur, vise précisément à produire un kaléidoscope d'images (remémorées, rêvées ou fantasmées) constituant autant d'instantanés de conscience.

This article deals with the reformulation devices at work in the novel Jealousy by A. Robbe-Grillet. The first two sections of the article are devoted to the numerous micro-variations which occur in the description of two particular scenes (namely workmen restoring a bridge down in the valley, and protagonists having drinks on the terrace) and to the effects of those subtle textual deformations on a reader looking for referential coherence. The third section focuses on the writing strategy of the author, which partakes in the "Nouveau Roman" trend; it is shown that the constant distortions/bifurcations, which confuse the reader's spatio-temporal landmarks, are precisely intended to build up kaleidoscopic images (whether memories, dreams or fantasies) amounting to momentary states of consciousness.

\section{INDEX}

Keywords : variant, repetition, bifurcation, distortion, space, perception, memory, fantasy, image, Nouveau Roman

Mots-clés : variante, répétition, bifurcation, déformation, espace, perception, souvenir, fantasme, image, Nouveau Roman

\section{AUTEUR}

CATHERINE FUCHS

CNRS, ENS, Université Sorbonne Nouvelle-Paris 3, LATTICE, UMR 8094, F-92120, France 